\title{
Manejo quirúrgico del quiste adrenal gigante: Reporte de caso y revisión de la literatura
}

\author{
William Jiménez R. ${ }^{1}$, Manuel Mosquera ${ }^{1}$, Karen Moreno $^{1}$ y Jacqueline Mugnier ${ }^{1}$
}

'Fundación Cardioinfantil. Instituto de Cardiología, Bogotá, Colombia.

Recibido el 2 de abril de 2018 aceptado para publicación el 16 de julio de 2018.

Correspondencia a: Dr. William Jiménez R. w.jimenez.md@gmail.com

\section{Surgical management of giant adrenal cyst: Case report and review of the literature}

Objective: Present a giant adrenal cyst (AC) case treated with minimally invasive resection and to perform a narrative literature review available. Material and Methods: A 54 year-old male presents with a left retroperitoneal slow growing mass, no symptoms, with a complex AC evidenced by previous images and mass biopsy, with suspected renal infiltration. A transabdominal laparoscopic resection is indicated. Results: A complex hemorrhagic $9 \mathrm{~cm}$ diameter AC was found, with adhesions to left Gerota's fascia. Complete resection of the AC was achieved through minimally invasive approach. The patient had an uneventful clinical recovery and was discharged on the second postoperative day. On 19th month of follow-up is completely asymptomatic. Even though the AC are benign lesions, the symptomatic giant AC, with fast growing ratio, and/or hemorrhagic conversion could be resected though laparoscopic adrenalectomy, with no increased morbidity or mortality. Discussion: The laparoscopic approach for giant non-functional AC should be considered as the standard of care. More evidence is required in terms of surgical approach outcomes to define clear recommendations. Conclusion: This report adds to the actual evidence in terms of minimally invasive approach for hemorrhagic giant AC.

Key words: adrenal cyst; adrenalectomy; laparoscopy.

\section{Resumen}

Objetivo: Presentar un caso de quiste adrenal (QA) gigante resecado por vía mínimamente invasiva y realizar una revisión narrativa de la literatura disponible al respecto. Materiales y Métodos: Se presenta el caso de un paciente de sexo masculino de 54 años, con una masa retroperitoneal izquierda de crecimiento lento, asintomático, con imágenes y biopsia sugiriendo un QA complejo con sospecha de infiltración renal. Se indica una resección laparoscópica transabdominal. Resultados: Se encuentra como hallazgo intraoperatorio un QA complejo hemorrágico de $9 \mathrm{~cm}$ de diámetro, con adherencia a la fascia de Gerota izquierda, logrando exéresis completa del QA por mínima invasión. El paciente evolucionó satisfactoriamente y fue dado de alta al segundo día posoperatorio. Es asintomático en 19 meses de seguimiento. Si bien los QA son benignos, los QA gigantes con crecimiento rápido, sintomáticos o de conversión hemorrágica, pueden ser resecados por adrenalectomía laparoscópica sin aumento de morbimortalidad. Discusión: El abordaje laparoscópico de los QA no funcionales gigantes debe ser considerado como el manejo de elección. Es necesaria más evidencia sobre resultados en diferentes abordajes para generar recomendaciones claras. Conclusión: Este reporte se suma a la evidencia actual en cuanto al abordaje mínimamente invasivo para QA gigantes hemorrágicos.

Palabras clave: quiste adrenal; adrenalectomía; laparoscopia.

\section{Introducción}

El quiste adrenal (QA) tiene una prevalencia entre $0,064-0,18 \%{ }^{1}$. Aunque se presenta a cualquier edad tiene un aumento entre la tercera y sexta década de la vida. Corresponde usualmente a lesiones benignas, unilaterales y se diagnostican hasta en un $57 \%$ en pacientes asintomáticos ${ }^{1}$.

La historia natural del QA ha sido pobremente reportada en la literatura y la información es escasa en términos de manejo definitivo ${ }^{2}$. Es aceptado que para el correcto abordaje del QA, se requiere dife- 
renciarlo de otros incidentalomas y debe descartarse funcionalidad paraclínica ${ }^{3,4}$. Sin embargo, su indicación quirúrgica no es clara.

El objetivo de este estudio es presentar un caso de un paciente con QA gigante con resección por técnica mínimamente invasiva. Posteriormente, se describen las opciones terapéuticas actuales mediante una revisión narrativa de la evidencia científica para lograr una aproximación al mejor tratamiento actual disponible.

\section{Reporte de caso}

Un hombre de 54 años es remitido a consulta por 4 años de evolución de sensación de masa en cuadrante superior izquierdo, sin síntomas adicionales. Como único antecedente colecistectomía laparoscópica 3 meses antes de consultar. En la valoración física se encuentra paciente normotenso, el abdomen sin masa palpable en el cuadrante superior izquierdo. Los paraclínicos iniciales (hemograma, bioquímica sanguínea, función renal y tiempos de coagulación) fueron normales. Se encontraba en seguimiento imagenológico extrainstitucional por un QA asintomático; se descarta funcionalidad por paraclínicos. El TC abdominal inicial (junio de 2013) muestra una masa compleja quística retroperitoneal izquierda, asociada a la glándula suprarrenal del mismo lado, de dimensiones en corte axial de diámetro anteroposterior (DAP) y diámetro lateral (DL) de $45 \mathrm{~mm}$ x $46 \mathrm{~mm}$, respectivamente, sugestiva de quiste adrenal benigno (Figura 1-A); y sucesivos controles escanográficos que se presentan en las imágenes (Figura 1-B, 1-C, 1-D), con seguimiento que se resume en la Figura 1. La ecografía de abdomen muestra una lesión quística retroperitoneal izquierda, con múltiples septos en su interior, con dimensiones de 68 x 72 x $58 \mathrm{~mm}$. La ecoendoscopia concluye una lesión quística adyacente a la cola del páncreas de $64 \mathrm{~mm}$ x $55 \mathrm{~mm}$.
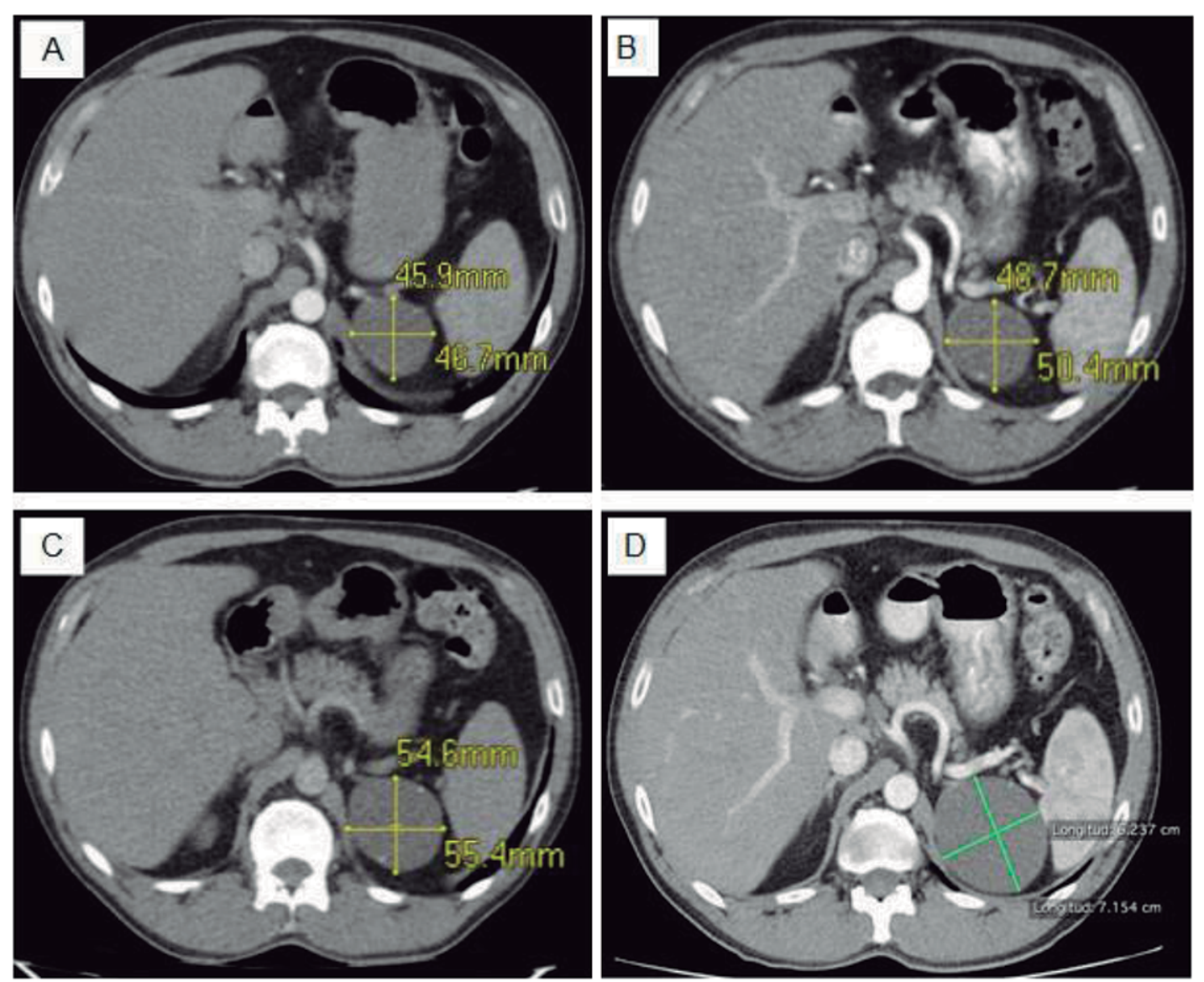

Figura 1. Evolución escanográfica del quiste adrenal (QA) en un periodo de 34 meses. A: Imagen inicial demostrando QA de $46 \times 45 \mathrm{~mm}$ tomada el 22/06/2013. B: Seguimiento imagenológico con crecimiento de $4 \mathrm{~mm}$ de diámetro lateral (DL) y $2 \mathrm{~mm}$ de diámetro anteroposterior (DAP), tomada el 11/02/2014. C: Crecimiento de QA muestra incremento de $5 \mathrm{~mm}$ de $\mathrm{DL}$ y $6 \mathrm{~mm}$ DAP, tomada el 23/10/2014. D: Último TC prequirúrgico, evidenciando crecimiento de QA con crecimiento DL $7 \mathrm{~mm}$ adicional, y DAP $17 \mathrm{~mm}$ tomada el 24/05/16. 

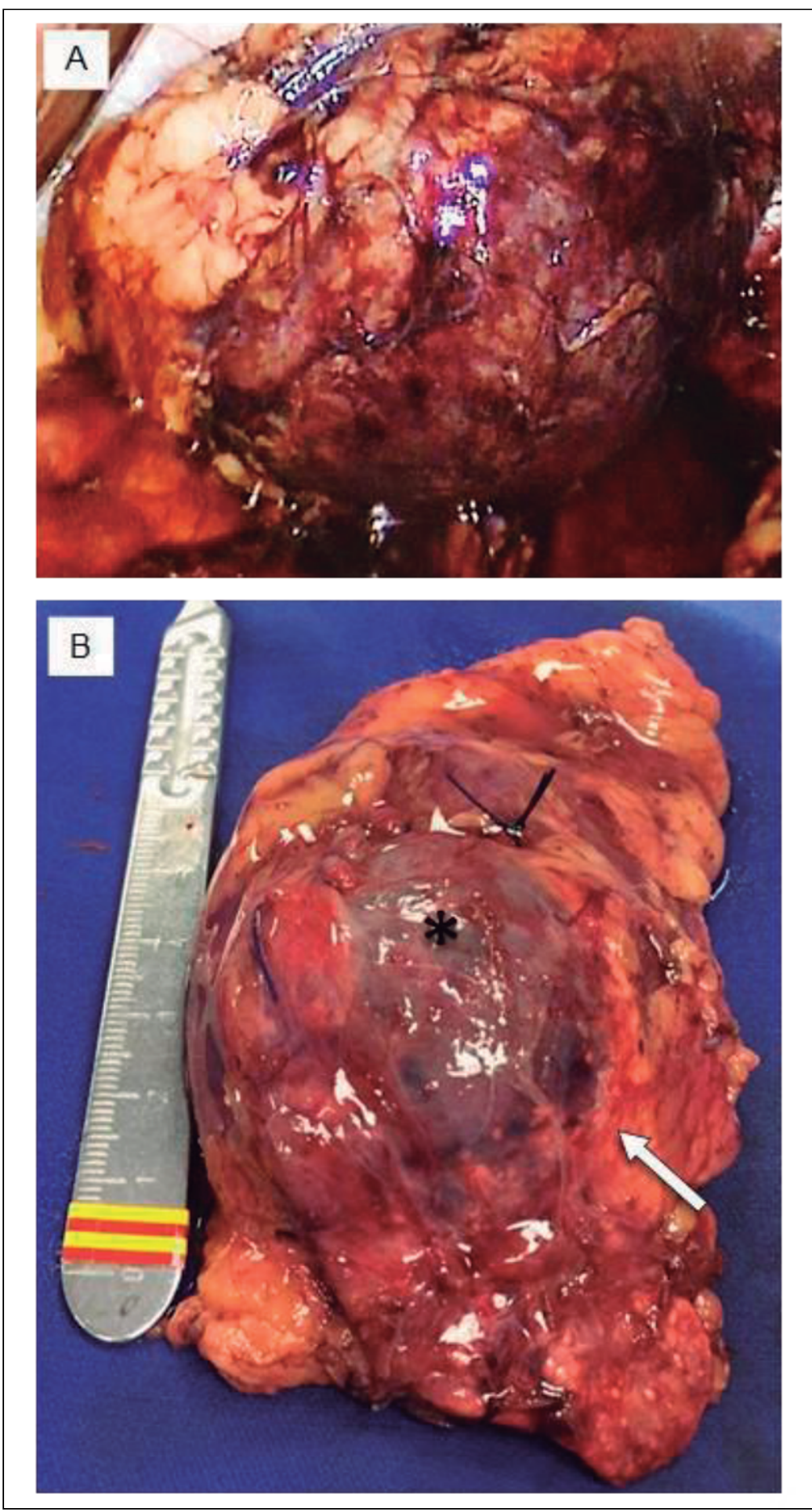

Figura 2. Hallazgos intraoperatorios de quiste adrenal (QA) durante adrenalectomía laparoscópica. A: Imagen mostrando el quiste adrenal (QA), disecado en posición anatómica dentro de la cavidad abdominal, altamente vascularizado, involucrando fascia de Gerota, rodeado de tejido glandular suprarrenal escaso. B: Espécimen quirúrgico resecado en mesa de mayo. Seda marcando porción supero-anterior del QA. Obsérvese el contenido hemorrágico (asterisco), y la distribución parcial de glándula suprarenal (flecha).
Se considera que la lesión corresponde a un QA no funcional, sin embargo, se cuestiona malignidad ante septos y estrecho contacto con riñón izquierdo. Se realiza una biopsia percutánea guiada por $\mathrm{TC}$, obteniendo líquido xantocrómico, y la patología confirmó un QA complejo, sin células malignas.

Se discute el caso en junta del Servicio de Cirugía General en conjunto con los Servicios Radiología y Urología concluyendo que el QA complejo izquierdo no muestra plano de clivaje claro con el riñón. Adicionalmente, presenta crecimiento imagenológico de $25 \mathrm{~mm}$ en 34 meses. Ante la incertidumbre diagnóstica y sospecha de invasión por vecindad e incremento progresivo de tamaño se decide resección quirúrgica.

Se realiza la adrenalectomía laparoscópica transperitoneal en decúbito lateral con 4 puertos, encontrando un QA de $9 \mathrm{~cm}$ de diámetro, complejo, multitabicado, de contenido hemorrágico, con escaso tejido glandular periférico (Figura 2). Se reseca parcialmente la cápsula de gerota por adherencia. La pieza quirúrgica se extrae en bolsa de vaiflex ampliando el puerto del flanco izquierdo. No se dejaron drenes. No hubo complicaciones ni sangrado.

El paciente recibió dieta líquida el mismo día de la cirugía y analgesia sin opiáceos. Se recomendó deambulación temprana y se aseguró terapia respiratoria con incentivo. El proceso de recuperación fue satisfactorio, se avanzó dieta, se tornó asintomático y egresó al segundo día posoperatorio. La histopatología concluyó tejido de glándula suprarrenal con un pseudoquiste adrenal, sin hallazgos que sugieren malignidad (Figura 3), con citología intraquística proteinacea sin evidencia de células malignas.

En control los 19 meses del egreso el paciente se encuentra, asintomático y sin evidencia de recidiva en TC de control.

\section{Discusión}

Es preciso conocer el comportamiento del QA para definir su abordaje terapéutico. La clasificación del QA no funcional se ha realizado a partir de su histopatología en endotelial, pseudoquístico, epitelial o parasítico, en su mayoría benignos ${ }^{5-7}$. Los más frecuentes son los endoteliales $(45 \%)$, variedades linfoangiomatoso o angiomatoso, los marcadores inmunohistoquímicos sugieren origen vascular ${ }^{8}$, seguidos por los pseudoquísticos $(39 \%)^{6}$. Otros autores reportan con más frecuencia los pseudoquísticos $(62,9 \%)^{9}$, con una propensión mayor a malignizar$\mathrm{se}^{10}$. La conversión a malignidad global reportada, independientemente su histología, es del $7 \%{ }^{11}$. La 
histopatología del caso presentado concluyó que se trata de un pseudoquiste complejo (tabicaciones), pero sin hallazgos de malignidad (Figura 3).

La literatura disponible no es concluyente sobre el comportamiento biológico de los $\mathrm{QA}^{10,12}$, lo que limita su enfoque. Se mantiene la clasificación histopatológica planteada hace aproximadamente 60 años $^{6}$. La limitante sobre la indicación quirúrgica desde el punto de vista histopatológico radica en que la histopatología definitiva se obtiene con el análisis de la pieza quirúrgica. Esto imposibilita considerar esta variable para definir el manejo en QA no funcionales.

Además, del potencial de malignización, se han reportado otras indicaciones quirúrgicas en QA no funcionales, incluyendo: el crecimiento del QA, la hemorragia con colapso cardiovascular, la ruptura quística, o el compromiso de órganos vecinos con incertidumbre etiológica ${ }^{1,2,13-15}$. En este caso se descartó funcionalidad, hubo crecimiento quístico, hallazgos de complejidad e incertidumbre etiológica con sospecha de compromiso renal, que no se aclaró con imágenes (ecografía, TC de abdomen y ecoendoscopia), por lo que se resecó.

El tamaño y el crecimiento del QA han sido unos de los principales criterios quirúrgicos. Si bien imagenológicamente se han reportado incrementos del diámetro del QA hasta en un $60 \%$ de todos los casos, este crecimiento no se ha relacionado específicamente a malignización o complicaciones quísticas $^{2}$. Sin embargo, se ha indicado iniciar manejo en QA no funcionales cuando superan los $6 \mathrm{~cm}$ de diámetro ${ }^{2}$, por considerarse gigantes. En cuanto al crecimiento existen reportes de aumento del diámetro quístico hasta de $15 \mathrm{~cm}$ en 6 meses $^{16}$, lo cual puede estar asociado a hemorragia intraquística ${ }^{16}$. El caso reportado en este trabajo presentó un crecimiento de $2,5 \mathrm{~cm}$ en 34 meses sustentando la decisión quirúrgica, con hallazgo de quiste hemorrágico intraoperatorio lo que sugiere que el crecimiento quístico puede deberse a su conversión hemorrágica, asociación no estudiada aún.

Cabe recordar que los QA presentan una alta vascularización dependiente de la glándula suprarrenal, lo cual infiere el riesgo de complicación hemorrágica. Los QA con hemorragia intraquística o ruptura con repercusión hemodinámica son una urgencia quirúrgica $^{17}$, lo cual cuestiona la pertinencia de estudios invasivos que puedan convertir QA simples en hemorrágicos. En este caso se realizó punción diagnóstica ante la sospecha de malignidad. Dicha punción descartó células tumorales, lo que permitió considerar otras opciones terapéuticas a la cirugía como escleroterapia. Los hallazgos intraoperatorios como la patología final evidenciaron hemorragia antigua, siendo probable su conversión hemorrágica autolimitada pospunción, situación no estudiada en la actualidad, pero factible. La tendencia en el manejo de los QA es hacia la resección quirúrgica electiva una vez diagnosticado y descartada funcionalidad $^{18}$. Se ha reportado previamente que en los QA gigantes o complejos debe procederse a resección quirúrgica con principios oncológicos ${ }^{19}$, sin punción preoperatoria. Sin embargo, se ha evidenciado una tendencia a punciones diagnósticas por sospecha imagenológica de malignidad ${ }^{2}$.
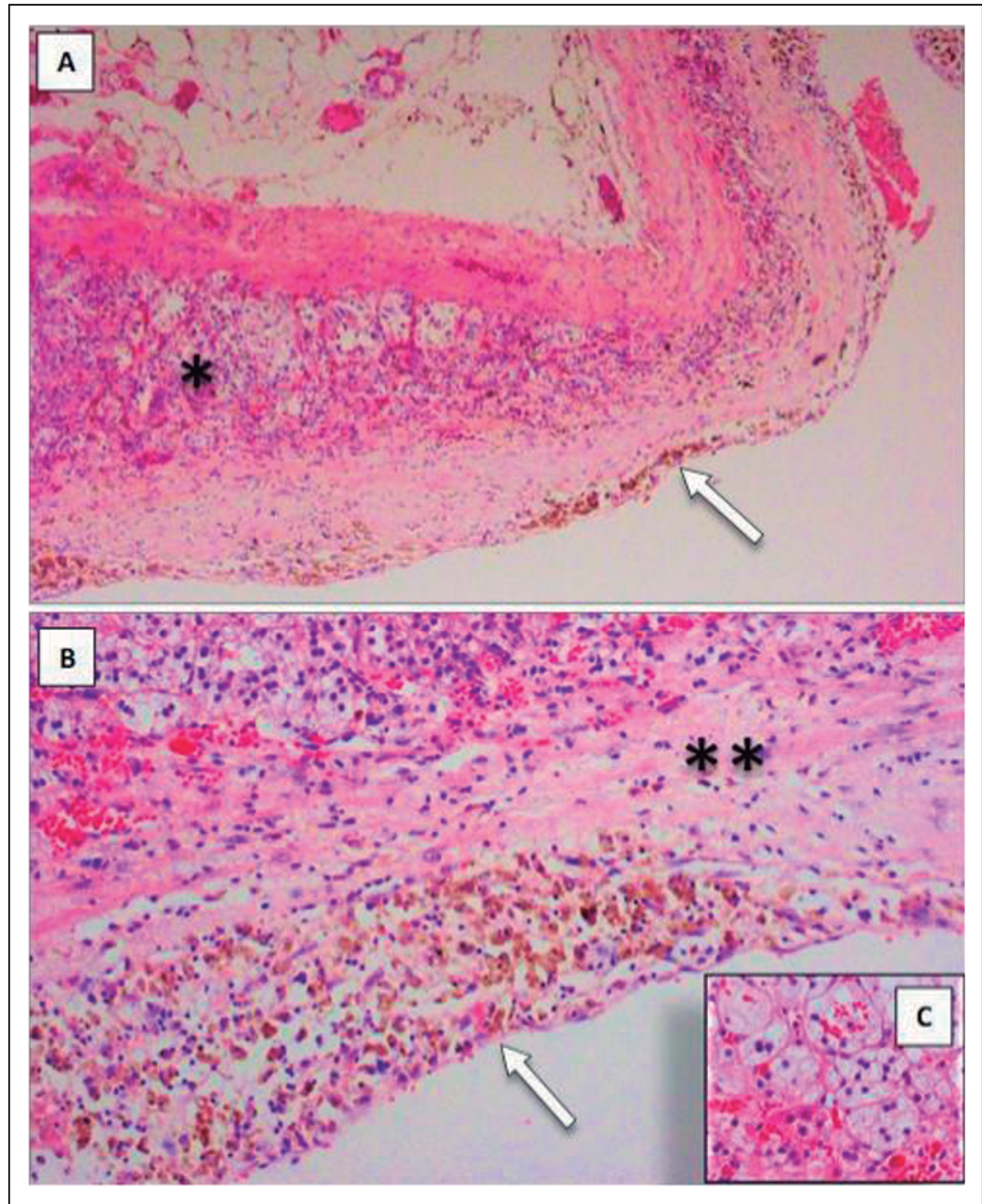

Figura 3. Resultado de histopatología del pseudoquiste adrenal. Coloración H+E. A: (4X) Hallazgos histopatológicos sobre pared del pseudoquiste suprarenal, con pared interna mostrando fibrosis y hemorragia antigua hacia la luz quística (flecha). Tejido cortical suprarrenal usual hacia pared (asterisco). B: (10X) Hemorragia antigua en pared interna quística (flecha). Tejido fibrótico circundante con células de inflamación (doble asterisco). C: Aumento 40X. Células corticales suprarrenales de características usuales, de citoplasma amplio, claro, con contenido lipídico, dispuestas en nidos redondeados, con septos finos en su interior. 
En cuanto al abordaje quirúrgico, la literatura reporta menor proporción de abordajes abiertos transabdominales para la resección de QA, con preferencia por el abordaje laparoscópico como técnica segura ${ }^{20}$, sin impacto en morbilidad o morta$\operatorname{lidad}^{15,18,21}$. La laparoscopia ha permitido desarrollar variaciones a la adrenalectomía convencional en la resección del QA en pacientes asintomáticos, como la decorticación y marzupialización quística laparoscópica, la cistectomía laparoscópica con preservación de la glándula suprarenal o la adrenalectomía laparoscópica parcial ${ }^{14}$.

Estudios previos demuestran múltiples beneficios de la mínima invasión en resección de QA no funcionales. Cavallaro, et al. ${ }^{13}$ muestra su experiencia en Italia en el manejo quirúrgico de lesiones adrenales quísticas, abogando por la resección laparoscópica lateral transabdominal por encima de la resección abierta, demostrando que la laparoscopía disminuyó el tiempo quirúrgico (92 a $86 \mathrm{~min}$ ), y la estancia hospitalaria (de 7,5 a 3,2 días) ${ }^{13}$. Estos resultados coinciden con los presentados por Lyu, et al. ${ }^{1}$ quien reporta su experiencia en China, con diferencias significativas en cuanto a tiempo quirúrgico entre cirugía abierta vs laparoscópica: $95 \mathrm{vs}$ 62 minutos, respectivamente $(\mathrm{p}<0,001)$, y menor tiempo de hospitalización en manejo laparoscópico sobre cirugía abierta: 4,47 vs 6,09 días, respectivamente $(\mathrm{p}=0,006)^{1}$. En el presente caso, el paciente fue dado de alta dos días después del procedimiento, un tiempo menor al reportado en estudios previos. Igualmente, la laparoscopia reduce las pérdidas sanguíneas intraoperatorias y mejora los resultados cosméticos comparativamente con cirugía abierta ${ }^{15}$. Esta evidencia fortalece el manejo laparoscópico sobre el abierto en los QA.

Por otra parte, el tamaño del QA no es determinante para definir abordaje abierto o laparoscópico. La literatura reporta QA entre 3,4-18 cm, sin embargo, no hay diferencias en cuanto a tamaño y éxito operatorio comparando abordaje abierto $v s$ laparoscópico ${ }^{15}$. Por esta razón y considerando los beneficios de la mínima invasión, se debe ofrecer manejo quirúrgico laparoscópico a todo paciente con QA.

Alternativamente, existen informes sobre el drenaje percutáneo del QA benigno como opción terapéutica, indicando cirugía únicamente en casos de recidiva ${ }^{5}$. La escleroterapia percutánea ablativa con etanol al 95\%, reporta casos en la literatura de éxito en pacientes con recidiva de QA posdecorticación laparoscópica ${ }^{22}$. Sin embargo, la tendencia es hacia la resección quirúrgica sin procedimientos percutáneos de drenaje prequirúrgico, con mejores resultados ${ }^{13}$. La recidiva del QA se reporta en $32 \%$ con drenaje percutáneo ${ }^{5}$, comparada con $0-2,1 \%$ con manejo quirúrgico ${ }^{1,13,15}$. No hay evidencia en la literatura comparando los resultados a corto o largo plazo de estas técnicas, y es claro que el drenaje percutáneo se considera una opción terapéutica en desuso ${ }^{2}$.

Cabe anotar que, tanto la escleroterapia percutánea con etanol como el drenaje simple, son herramientas terapéuticas válidas indicadas en pacientes con QA no funcional de alto riesgo quirúrgico, o que rechacen el procedimiento quirúrgico ${ }^{22}$. El paciente narrado está en su decimonoveno mes de seguimiento posoperatorio sin recurrencia acorde con la literatura. Nuestro grupo considera las opciones de drenaje percutáneo insuficientes para el tratamiento definitivo del QA, debido a su alta tasa de recidiva e inefectividad. Estas formas se alzan como alternativa diagnóstica ante duda neoplásica, o como alternativa terapéutica en pacientes con claras contraindicaciones quirúrgicas (condición médica o rechazo del paciente). De lo contrario, nuestro grupo propende por la resección quirúrgica del QA, idealmente laparoscópica, en casos de crecimiento rápido, sospecha de malignidad o descompensación clínica y sintomatológica.

Concluyendo, el abordaje terapéutico de los QA no funcionales gigantes debe basarse en los hallazgos imagenológicos sugestivos de malignidad, complejidad o con alta tasa de crecimiento. Se indica punción diagnóstica en QA gigantes ante sospecha de malignidad, y se indica resección quirúrgica idealmente por laparoscopia como técnica segura en paciente estable independientemente el tamaño del QA.

\section{Conflictos de interés}

Sin conflictos de interés. 


\section{Referencias}

1. Lyu X, Liu L, Yang L, Gao L, Wei Q. Surgical management of adrenal cysts: A single-institution experience. Int Braz J Urol. 2014;40:656-65.

2. Ricci Z, Chernyak V, Hsu K, Mazzariol FS, Flusberg M, Oh S, et al. Adrenal cysts: Natural history by long-term imaging follow-up. Am J Roentgenol. 2013;201:1009-16.

3. Herrera MF, Grant CS, van Heerden JA, Sheedy PF, Ilstrup DM. Incidentally discovered adrenal tumors: an institutional perspective. Surgery. 1991;110:1014-21.

4. Grumbach MM, Biller BMK, Braunstein GD, Campbell KK, Carney JA, Godley PA, et al. Management of the clinically inapparent adrenal mass ("incidentaloma"). Ann Intern Med. 2003;138:424-9.

5. Neri LM, Nance FC. Management of adrenal cysts. Am Surg. 1999;65:15163.

6. Foster DG. Adrenal cysts. Review of literature and report of case. Arch Surg. 1966;92:131-43.

7. Abeshouse GA, Goldstein RB, Abeshouse BS. Adrenal cysts; review of the literature and report of three cases. J Urol. 1959;81:711-9.

8. Janevska V, Janevski V, Stankov O, Spasevska L, Kostadinova-Kunovska S, Zhivadinovik J. Non-Tumor Cystic Lesions of the Adrenal Gland. PRILOZI. 2015;36. doi:10.1515/prilozi-2015-0078.
9. Tanuma Y, Kimura M, Sakai S. Adrenal cyst: a review of the Japanese literature and report of a case. Int J Urol. 2001;8:500-3.

10. Chien H-P, Chang Y-S, Hsu P-S, Lin JD, Wu YC, Chang HL, et al. Adrenal Cystic Lesions: A Clinicopathological Analysis of 25 Cases with Proposed Histogenesis and Review of the Literature. Endocr Pathol. 2008;19:274-81.

11. Major P, Pędziwiatr M, Matłok M, Ostachowski M, Winiarski M, Rembiasz $\mathrm{K}$, et al. Cystic adrenal lesions-analysis of indications and results of treatment. Pol Przegl Chir. 2012;84:184-9.

12. Koperski $Ł$, Pihowicz P, Szczepankiewicz B, Fus L, Cyran A, Bogdanska $\mathrm{M}$, et al. Clinicopathological and immunohistochemical analysis of epithelial-lined (true) cysts of the adrenal gland with proposal of a new histogenetic categorization. Pathol Res Pract. 2017;213:1089-96.

13. Cavallaro G, Crocetti D, Paliotta A, De Gori A, Tatallo MR, Letizia C, et al. Cystic adrenal lesions: Clinical and surgical management. The experience of a referral centre. Int J Surg. 2015;13:23-6.

14. Castillo OA, Litvak JP, Kerkebe M, Urena RD. Laparoscopic management of symptomatic and large adrenal cysts. J Urol. 2005;173:915-7.

15. El-Hefnawy AS, El Garba M, Osman Y, Eraky I, El Mekresh M, Ibrahim E-H. Surgical management of adrenal cysts: single-institution experience. BJU Int. 2009;104:847-50.

16. Poiana $\mathrm{C}$, Carsote $\mathrm{M}$, Chirita $\mathrm{C}$, Terzea D, Paun S, Beuran M. Giant adrenal cyst: case study. J Med Life. 2010;3:308-13.

17. Chew SP, Sim R, Teoh TA, Low CH. Haemorrhage into non-functioning adrenal cysts-report of two cases and review of the literature. Ann Acad Med Singapore. 1999;28:863-6.

18. Pradeep P V, Mishra AK, Aggarwal V, Bhargav PRK, Gupta SK, Agarwal A. Adrenal cysts: an institutional experience. World J Surg. 2006;30:1817-20.

19. Hung S-F, Chung S-D, Chueh S-C, Lai $\mathrm{M}-\mathrm{K}$, Yu H-J. Laparoscopic Management of Potentially Malignant or Complex Adrenal Cysts Abiding by the Principles of Surgical Oncology. J Endourol. 2009;23:107-14.

20. Pogorzelski R, Toutounchi S, Krajewska E, Fiszer P, Pachucki J, Bednarczuk T, et al. Laparoscopic treatment of adrenal cysts-own research and literature review. Endokrynol Pol. 2015;66:469-72

21. Kodama K, Takase Y, Niikura S, Shimizu A, Tatsu H, Saito K. Laparoscopic Management of a Complex Adrenal Cyst. Case Rep Urol. 2015;2015:1-4.

22. Hatzidakis A, Kozana A, Petrakis I, Mamoulakis C. Postsurgical large adrenal cyst recurrence: treatment by means of percutaneous alcohol ablation. BMJ Case Rep. 2014;2014:bcr2014206962bcr2014206962. doi:10.1136/bcr-2014206962 . 